\title{
Multi-variable Constrained Control Approach for a Three-Dimensional Eel-like Robot
}

\author{
M. El Rafei, M. Alamir, N. Marchand, M. Porez and F. Boyer
}

\begin{abstract}
In this paper, a multi-variable feedback design for the 3D movement of an eel-like robot is presented. Such a robot is under construction in the context of a national French robotic project. The proposed feedback enables the tracking of a desired 3D position of the eel's head as well as the stabilization of the rolling angle. The control design is based on a recently developed reduced model that have been validated using a 3D complete continuous model described in [3]. Several scenarios are proposed to assess the efficiency of the proposed feedback law.
\end{abstract}

\section{INTRODUCTION}

In this paper, current researches on the control of an eel-like robot are presented. This work is carried out in the context of a multidisciplinary French national research project ${ }^{1}$. The aim of this project is to design, construct and control the 3D motion of an eel-like robot. The prototype under construction is obtained by connecting many parallel platforms (see figure 1). The eel's body will then be covered by a deformable "skin" in order to achieve high performance swimming. As it has been underlined by many researchers in this field, understanding the dynamics of such robots may be of a great interest in improving the manoeuvrability of under-water vehicles [15], [8], [11], [9], [6], [12], [14], [2].

A 3D continuous model of the target prototype has been proposed in [3] using the geometrically exact theory of beams under finite deformations [13]. This model that is not suitable for use in advance control derivation was used to identify and validate a low dimensional and computationally efficient reduced mean model that can be used in advanced control design. This 3D reduced model is clearly used here to design and validate the proposed $3 D$ control feedback. However, for the lack of space, the reduced model derivation is not treated by this paper. Nevertheless, it is still briefly described in section II.

There exist many works in the literature that studied the eel-like robots movements. In [9] and the related works, the 2D movement of an eel-like robot has been studied. The rolling cart analogy is used in order to derive state

This work was supported by the French National Center for Scientific Research (CNRS) in the context of the ROBEA-project and the French National research agency project (ANR-RAAMO).

M. El Rafei, M. Alamir and N. Marchand are with the control system department of GIPSA-Lab maher.el-rafei@lag.ensieg.inpg.fr, mazen.alamireinpg. fr, nicolas.marchandeinpg.fr

M. Porez and F. Boyer are with the Institut de Recherche en Cybernétique de Nantes. frederic.boyer@emn.fr, mathieu.porezeirccyn.ec-nantes.fr

${ }^{1} \mathrm{http} / / / \mathrm{www}$.irccyn.ec-nantes.fr/hebergement/ROBEA/ feedback that tracks some reference trajectory. Another interesting approach was proposed in [10], [11], [16] where averaging formulas have been derived to describe the mean behavior over an undulatory cycle. A design procedure for a biomimetic robot-fish based on improved kinematic propulsive model has been described in [17] where the basic motion control laws were presented. For a detailed review of existing works on the mechanics and the control of swimming, the reader can refer to [6]. However, few research have been

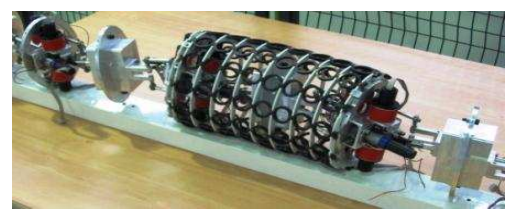

Fig. 1. The experimental assembly (under construction)

conducted on the control design for 3D motion of the eellike robots. Results on the 3D control of an eel-like robot are presented in [1] and [7] where a complete control scheme for 3D movement of the continuous model [3] was proposed. The motion and the velocity in the transverse plane are controlled by monitoring the oscillatory gait characteristics while the altitude changes and the rolling stabilization task are handled by means of two pectoral fins that are attached to the eel's head [1] or by 3D robot's body movements without using pectorals fins [7]. These decoupled control laws suffer however from a high sensitivity to the choice of the control parameters. That is why a coupled multi-variable control are still to be developed.

In this work, a coupled control scheme for 3D movement of the robot's reduced model is proposed. Basically, given the robot state and the targeted position, a desired velocity is computed that enables to incrementally achieve the tracking mission. This desired velocity is then used to build a constraint quadratic optimization problem in which, the decision variable are incremental actions to be taken over the current sampling period. This optimization problem is used also to achieve roll angle stabilization.

This paper is organized as follows : First, the reduced model is summarized in section II. Section III clearly states the $3 \mathrm{D}$ control problem. The control strategy is then presented in section IV, namely, the tracking of a desired 3D position as well as the rolling angle stabilization. Some $3 D$ scenarios are proposed in section V. The paper ends by some concluding remarks together with the road map for future works. 


\section{THE EEL-LIKE ROBOT REDUCED MODEL}

This section presents a simplified dynamic model for the 3D Eel-like robot. This model is used for the control purpose and it is based on the continuous model [3]. It consists in modeling the eel's head linear and angular mean velocities as dynamical functions of the control input. Figure 2 illustrates the basic notations used in the description of the model where the following notations are used :

- $\left(0, E_{1}, E_{2}, E_{3}\right)$ denotes the earth frame assimilated to a galilean reference.

- $\left(0, t_{10}, t_{20}, t_{30}\right)$ refers to the mobile frame attached to the eel's head.

- $t_{10}, t_{20}$ and $t_{30}$ are respectively the head's roll, pitch and yaw axes.

- $V_{0}=\left(\begin{array}{lll}V_{1} & V_{2} & V_{3}\end{array}\right)$ denotes the mean linear velocity of the eel's head expressed in the head frame. Moreover $V_{0}=\frac{1}{T} \int_{t-T}^{t}\left\|V_{0}(\tau)\right\| d \tau$

- $T$ is the undulation period. Note that the 3D control of the eel-like robot is achieved here without use of pectoral fins through the deformations of the end part of the deformable body [7]. The key idea consists in applying torsion and pitch movements to the tailing part of the body (see figure 3) in phase with its undulatory movement in order to generate pressure wave and fluid forces that are used by the control law.

- $w_{0}=\left(\begin{array}{lll}w_{p} & w_{q} & w_{r}\end{array}\right)$ denotes the mean rotation vector (angular velocity) of the head. Moreover $w_{0}=$ $\frac{1}{T} \int_{t-T}^{t}\left\|w_{0}(\tau)\right\| d \tau$

- $w_{p}, w_{q}$ and $w_{r}$ are respectively the head's roll, pitch and yaw mean velocities.

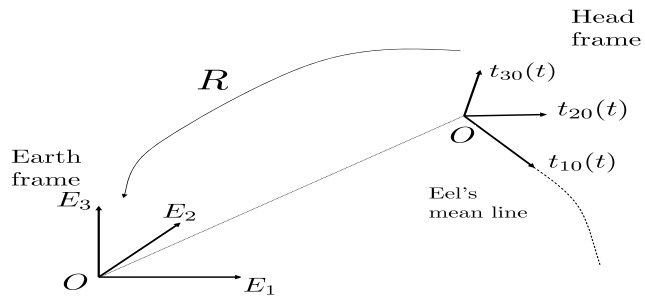

Fig. 2. Frames and parametrization of the reduced eel-like robot model

Our experience based on the continuous model [3] suggests to use the following structure for the reduced mean model :

$$
\begin{aligned}
\dot{V}_{1} & =-\lambda_{1}\left(u_{3}, u_{2}\right)\left[V_{1}-V_{1}^{\infty}\left(u_{3}, u_{2}\right)\right] \\
\dot{V}_{2} & =0 \\
\dot{V}_{3} & =-\lambda_{3}\left(u_{q}, V_{1}\right)\left[V_{3}-V_{3}^{\infty}\left(u_{q}, V_{1}\right)\right] \\
\dot{w}_{p} & =-\lambda_{p}\left(u_{p}, V_{1}\right)\left[w_{p}-w_{p}^{\infty}\left(u_{p}, V_{1}\right)\right] \\
\dot{q}_{1} & =-\lambda_{1 q}\left(u_{q}, V_{1}\right) q_{1}-\lambda_{2 q}\left(u_{q}, V_{1}\right) u_{q} \\
\dot{w}_{q} & =\left(1-2 \mu_{q}\left(u_{q}, V_{1}\right)\left|q_{1}\right|\right) \dot{q}_{1} \\
\dot{w}_{r} & =\rho \dot{V}_{1}-\lambda_{r} V_{1}\left(\rho-u_{1}\right) \\
\dot{\rho} & =-\lambda_{r}\left(\rho-u_{1}\right) \\
\dot{Q} & =\frac{1}{2} M(w) Q
\end{aligned}
$$

$$
\left(\begin{array}{c}
\dot{x} \\
\dot{y} \\
\dot{z}
\end{array}\right)=R_{q}(Q)\left(\begin{array}{c}
V_{1} \\
0 \\
V_{3}
\end{array}\right)
$$

where :

- $q_{1}$ is an internal variable.

- $\rho$ is the eel's body curvature. Note that the control law applies a uniform additional curvature (uniform along the body but variable in time) that is added to the non uniform curvature needed to enhance the undulation wave.

- $w_{r}=\rho V_{1}$.

- $Q=\left(\begin{array}{llll}q_{0} & q_{x} & q_{y} & q_{z}\end{array}\right)$ is the quaternion that represents the head frame's orientation with respect to the inertial frame. We can also represent this orientation by a rotation matrix $R_{q}(Q)$. The quaternion (and its time derivative) can be related to the rotation vector $w$. This relation (9) enables to have $Q$ as a function of $w$. For more informations about quaternion related formalism, the reader can refer to [5].

- $M(w)=\left(\begin{array}{cccc}0 & -w_{p} & -w_{q} & -w_{r} \\ w_{p} & 0 & w_{r} & -w_{q} \\ w_{q} & -w_{r} & 0 & w_{p} \\ w_{r} & w_{q} & -w_{p} & 0\end{array}\right)$ is a skewsymmetric tensor.

- $O(x, y, z)$ represents the $3 \mathrm{D}$ coordinates of the eel's head.

- $\mathbf{u}=\left(u_{p}, u_{q}, u_{1}, u_{2}, u_{3}\right)$ is the control input (see section III for more details).

- $\lambda_{1}, V_{1}^{\infty}, \lambda_{3}, V_{3}^{\infty}, \lambda_{p}, p^{\infty}, \lambda_{1 q}, \lambda_{2 q}, \mu_{q}$ are the identified parameters as functions of the control vector $\mathbf{u}$ and the leading velocity component $V_{1}$.

- $\lambda_{r}$ is a fixed constant parameter that directly monitors the body curvature.

This model is used here to derive the coupled 3D control for the robot's 3D motion.

\section{STATEMENT OF THE CONTROL PROBlEM}

Recall that the reduced model is identified and validated based on the complete continuous model presented in [3]. In the later, the robot is viewed as a beam defined by a continuous assembly of rigid cross sections. For this model a vector field $K(t, X)=\left(\begin{array}{lll}K_{1}(t, X) & K_{2}(t, X) & K_{3}(t, X)\end{array}\right)$ is the control input in which, $t$ is the time, $X$ designates the material abscissa along the eel's mean line. The last two components of $K$, namely $K_{2}$ and $K_{3}$ stand for the curvatures of the beam in the two planes $\left(G, t_{1}, t_{3}\right)(t, X)$ and $\left(G, t_{1}, t_{2}\right)(t, X)$ (see figure 3 ) while the first component $K_{1}$ stands for the torsion strain field. $G(X)$ the center of mass of the $X$ section.

As mentioned previously, the 3D control of the eellike robot is realized here by 3D robot's body movements without using its pectoral fins. For more informations about the without pectoral fins swimming feasibility problem, the reader can refer to [7].

The robot's back part can be defined by (See figure 3) :

$$
\chi_{b a c k}=\left[X_{b}, L\right]
$$


where $X_{b}$ is a given material abscissa and $L$ is the robot's length.

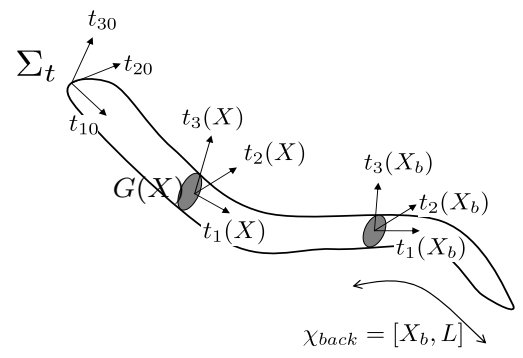

Fig. 3. Frames and parametrization.

The control law is based on the following predefined temporal structure of the undulation laws $K_{1}$ and $K_{2}$ :

$$
\begin{aligned}
\forall X \in \chi_{\text {back }}, \quad K_{1}(X, t) & =u_{q}(t) \cdot \cos \left(\frac{2 \pi}{T} t\right) \\
K_{2}(X, t) & =u_{p}(t) \cdot \cos \left(\frac{2 \pi}{T} t\right)
\end{aligned}
$$

where $u_{q} \in\left[-u_{q}^{\max }, u_{q}^{\max }\right]$ and $u_{p} \in\left[-u_{p}^{\max }, u_{p}^{\max }\right]$ are used as control variables. $u_{q}$ is the twist angle and $u_{p}$ is the pitch angle.

As for the undulation law $K_{3}$, the following structure is adopted in accordance with biological observations [4] :

$$
K_{3}(t, X):=u_{3}(t) \cdot A\left(X, u_{2}(t)\right) \sin \left(\frac{X}{\lambda}-\frac{t}{T}\right)+u_{1}(t),
$$

where $u_{3} \in\left[0, u_{3}^{\max }\right], u_{2} \in\{-1,1\}$, and $u_{1} \in\left[0, u_{1}^{\max }\right]$. The control input $u_{2} \in\{-1,1\}$ defines whether the amplitude of undulations is bigger at the eel's tail or the eel's head. This is used to enhance acceleration or deceleration according to the velocity related control requirements. When the undulation law (14) is used with $u_{1} \equiv 0$, a strait movement is asymptotically obtained while constant non vanishing values of $u_{1}$ asymptotically lead to circular trajectories.

Note that (12)-(13)-(14) define a finite dimensioned parametrization of the control input leading to the control vector

$$
\mathbf{u}:=\left(u_{p}, u_{q}, u_{1}, u_{2}, u_{3}\right)
$$

The controller has to appropriately modify the control vector in order to steer the head towards the desired 3D position as well as to realize the rolling angle stabilisation and the velocity control.

\section{THE PROPOSED FEEDBACK}

After Linearization and using a small sampling period $\tau_{s}$, the equations (1), (3), (4), (6) and (7) become :

$$
\begin{aligned}
\delta V_{1} & =-\lambda_{1} \tau_{s}\left[V_{1}-V_{1}^{\infty}\right] \\
\delta V_{3} & =-\lambda_{3} \tau_{s}\left[V_{3}-V_{3}^{\infty}\right] \\
\delta w_{p} & =-\lambda_{p} \tau_{s}\left[w_{p}-w_{p}^{\infty}\right] \\
\delta w_{q} & =-\left(1-2 \mu_{q}\left|q_{1}\right|\right)\left(\lambda_{1 q} q_{1}+\lambda_{2 q} u_{q}\right) \\
\delta w_{r} & =\rho \tau_{s}\left(\dot{V}_{1}-\lambda_{r} V_{1}\right)+\lambda_{r} \tau_{s} V_{1} u_{1}
\end{aligned}
$$

where for all variable $F, F(k)$ is a short notation for $F\left(k \tau_{s}\right)$ and $\delta F=F(k+1)-F(k)$.

Recall that $\lambda_{1}, V_{1}^{\infty}, \lambda_{3}, V_{3}^{\infty}, \lambda_{p}, p^{\infty}, \lambda_{1 q}, \lambda_{2 q}, \mu_{q}$ are the identified parameters as functions of the control vector $\mathbf{u}$ [see (15)]. Let :

$$
\begin{array}{r}
\delta=\left(\begin{array}{lllll}
\delta V_{1} & \delta V_{3} & \delta w_{p} & \delta w_{q} & \delta w_{r}
\end{array}\right)^{T} \\
=\left(\begin{array}{lllll}
\delta_{1} & \delta_{2} & \delta_{3} & \delta_{4} & \delta_{5}
\end{array}\right)^{T} \\
\delta \in \Delta\left(\tau_{s}, V_{1}, \mathbf{u}\right)=\left[\begin{array}{ll}
\delta_{\min }, \delta_{\max }
\end{array}\right]
\end{array}
$$

denotes the intermediate unknown vector that has to be computed in order to achieve the tracking objective. Once these increments are computed, this gives reference values for the lower level control vector $\mathbf{u}$. The later is then computed by a constrained optimization step. More precisely, having the robot's velocity $V_{A}(k)$ at a given instant $k$, the control strategy consists in the computation of the desired velocity $V_{A}^{d}(k+1)$ to reach at the next sampling time $(k+1)$. Once this desired velocity is computed, the increment $\delta V$ that is directly linked to the increment vector $\delta$ [through equations (16)-(17)] enables to derive an optimization problem in which the roll angle stabilization is also taken into account. The whole formalism is precisely given in the next sections.

The $\delta$ 's lower and upper bounds $\left(\delta_{\min }, \delta_{\max }\right)$ are computed on line according to the current robot's state. More precisely, at each sampling period, given the robot's state, the control vector saturation levels $\left(\mathbf{u} \in\left[\mathbf{u}_{\min }, \mathbf{u}_{\max }\right]\right)$ and the static maps of the different model's parameters (that are dependent of the control input), the $\delta$ 's lower and upper bounds are computed using the equations (16)-(20).

\section{A. Defining the desired velocity $V_{A}^{d}(k+1)$}

Let $P_{A}, V_{A}$ designate the robot's position and velocity at a given instant $k$ and $P_{C}$ the desired objective (see figure 4). $P_{A 0}^{+}, V_{A 0}^{+}$are respectively the position and velocity that would be obtained at the next sampling instant $(k+1)$ if $\delta(k)=0$ is applied during the sampling period.

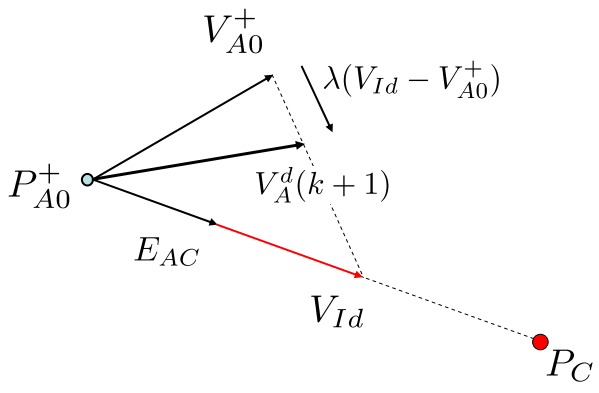

Fig. 4. Control strategy

Note that all quantities are expressed in the earth frame. The ideal velocity that can realize the robot's mission is 
directed by $E_{A C}$, where $E_{A C}=\frac{P_{A 0}^{+} P_{C}}{\left\|P_{A 0}^{+} P_{C}\right\|}$

More precisely, the ideal velocity would be given by :

$$
V_{I d}=\min \left(V_{\max }, \frac{\left\|P_{A 0}^{+} P_{C}\right\|}{\tau_{s}}, \sqrt{2\left\|P_{A 0}^{+} P_{C}\right\| \gamma_{d \max }}\right) E_{A C}
$$

since this takes into account the achievable maximum velocity $V_{\max }$, the sampling nature of the control law and the fact that one would like to reach the objective at zero velocity which imposes some deceleration margin that is compatible with the maximum deceleration module $\gamma_{d \max }$.

Now the ideal velocity is generally not achievable in within the actuator constraint, that is why an interpolation is introduced through the parameter $\lambda$ leading to the following desired velocity :

$$
V_{A}^{d}(k+1)=V_{A 0}^{+}+\lambda\left(V_{I d}-V_{A 0}^{+}\right)
$$

where $\lambda \in[0,1]$ is a parameter that is adapted on line according to the current configuration in order to tackle dynamically the actuator saturations. In this paper however, this parameter is determined through worst case calibration for simplicity.

\section{B. Velocity tracking related term}

The constrained optimization problem leading to the computation of $\delta$ is obtained via constrained trade-off between the velocity tracking consideration and the roll angle stabilization concern. In this section, the velocity tracking related term used in the optimization problem is derived. The robot velocity in the earth frame at an instant $k$ is given by the equation (10) :

$$
V_{A}(k)=R_{q}(Q(k))\left(\begin{array}{c}
V_{1}(k) \\
0(k) \\
V_{3}(k)
\end{array}\right)
$$

Simple computations show that as long as the linear approximation is used, the conditions of perfect tracking $\left.V_{A}(k+1)=V_{A}^{d}(k+1)\right)$ is equivalent to the following linear equation in the unknown vector $\delta$ :

$$
A \cdot \delta=B
$$

where :

$$
\begin{array}{r}
A=A\left(Q(k), V_{A}^{d}(k+1)\right) \\
B=B\left(Q(k), V_{A}^{d}(k+1), V_{0}(k)\right)
\end{array}
$$

in witch $A$ is a $(3 \times 5)$ matrix, $B$ is a $(3 \times 1)$ matrix and

$$
V_{0}(k)=\left(\begin{array}{c}
V_{1}(k) \\
V_{2}(k) \\
V_{3}(k)
\end{array}\right)
$$

is the robot velocity in the head mobile frame [see (1), (2) and (3)].

\section{Rolling angle stabilization related term}

The control of the rolling angle amounts to control $p_{\text {rol }}:=$ $t_{20} \cdot E_{3}$ around 0 . This amounts to control the head's roll velocity $w_{p}$ suggesting the following ideal relation :

$$
\dot{w}_{p}=-\gamma_{1}\left(w_{p}-w_{p}^{d}\right) \quad ; \quad w_{p}^{d}=-\gamma_{2} p_{\text {rol }}
$$

that can be written after Linearization as follows :

$$
\delta w_{p}=-\gamma_{1} \tau_{s}\left(w_{p}+\gamma_{2} p_{\text {rol }}\right)
$$

$\gamma_{1}$ and $\gamma_{2}$ are control parameters.

Here again, using the incremental equations (18)-(20), the above can be written in terms of the incremental vector $\delta$ in the following compact form :

$$
A_{1} \cdot \delta=B_{1}
$$

where :

$$
\begin{aligned}
& A_{1}=\left(\begin{array}{lllll}
0 & 0 & 1 & 0 & 0
\end{array}\right) \\
& B_{1}(k)=-\gamma_{1} \tau_{s}\left(w_{p}(k)+\gamma_{2} p_{\text {rol }}(k)\right)
\end{aligned}
$$

\section{Controller formulation}

Gathering together the two requirements, the following constrained optimization problem can be derived for the computation of $\delta$ :

$$
\begin{gathered}
\min _{\delta \in \Delta}\left(\eta \cdot \delta^{T} \delta+\alpha_{1}\|A \delta-B\|^{2}+\alpha_{2}\left\|A_{1} \delta-B_{1}\right\|^{2}\right) \\
\delta \in \Delta\left(\tau_{s}, V_{1}, \mathbf{u}\right)=\left[\delta_{\text {min }}, \delta_{\text {max }}\right]
\end{gathered}
$$

where :

- $\delta$ is the increment to be computed and $\mathrm{u}$ is the current control value. [see (15)]

- $\eta, \alpha_{1}, \alpha_{2}$ are control parameters.

- $\tau_{s}$ is the sampling period.

The first part of the equation (33) is a regulation term, the second part allows to take into account the velocity tracking while the third part accounts for the rolling angle stabilisation.

This optimization problem can be written as a time varying quadratic problem :

$$
\min _{\delta \in \Delta}\left(\frac{1}{2} \delta^{T} S \delta+f^{T} \delta\right)
$$

with the constraints :

$$
\delta_{\min } \leq \delta \leq \delta_{\max }
$$

where :

$$
\begin{aligned}
S & =2\left(\alpha_{1} A^{T} A+\eta E+\alpha_{2} A_{1}^{T} A_{1}\right) \\
f & =-2\left(\alpha_{1} A^{T} B+\alpha_{2} A_{1}^{T} B_{1}\right)
\end{aligned}
$$

\section{Simulations}

In this section, some numerical simulations are proposed to assess the efficiency and underline some interesting features of the proposed solution, using the reduced and the complete continuous model. 


\section{A. The robot parameters}

The exhaustive definition of the model parameters is given in [3]. Let us mention here that the length of the robot is $L=2.08 \mathrm{~m}$ and all the cross sections are ellipsoidal with evolutive dimension that reproduces a quite realistic and faithful form (the tail is thinner than the central body).

\section{B. Control related parameters}

- The sampling period $\tau_{s}=1.2 \mathrm{~s}$.

- $35 \%$ of the robot's length are used as the robot's back part that realize the pitch and the twist movements (swimming without pectoral fins)[see (11)].

- The control input $u_{3} \in[0,1.4]$ and $u_{2}=[-1,1]$ (see section III) : For $u_{3}=1.4$, we obtained a maximal velocity of $50 \mathrm{~cm} / \mathrm{sec}\left(u_{3}=1\right)$ in acceleration mode $\left(u_{2}=-1\right)$.

- The twist angle $u_{q} \in\left[-20^{\circ}, 20^{\circ}\right]$ (see section III).

- The pitch angle $u_{p} \in\left[-4^{\circ}, 4^{\circ}\right]$ (see section III).

- The maximal body curvature $u_{1}^{\max }=0.5$. $\left(u_{1} \in\left[0, u_{1}^{\max }\right]\right)$, see section III.

- $\lambda_{r}=1.0[$ see (8)].

- $\lambda=0.5$ [see (25)].

- $\gamma_{1}=1, \gamma_{2}=1$ [see (30)].

- $\alpha_{1}=1, \alpha_{2}=10^{-2}[\operatorname{see}(33)]$.

- $\eta=10^{-10}\left\|A^{T} A\right\|+10^{-6}$ [see (33)].

- $V_{\max }=40 \mathrm{~cm} / \mathrm{sec}[\operatorname{see}(24)]$.

\section{Manoeuvre description}

Two set-point changes are successively and simultaneously done on the three coordinates of the desired position $P_{d}$. The robot is initially at rest. The desired state is then defined by the following expression :

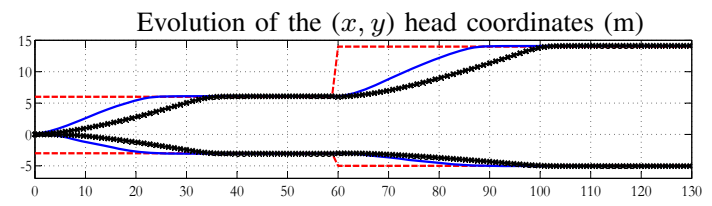

Evolution of the $(z)$ head coordinates $(\mathrm{m})$

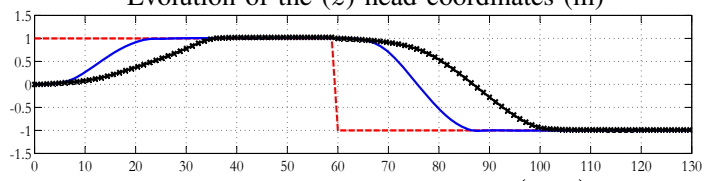

Evolution of the rolling angle $\arcsin \left(p_{\text {rol }}\right)(\mathrm{deg})$

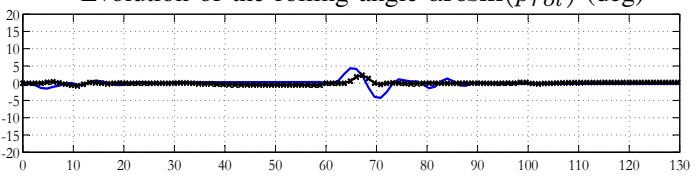

Time (seconds)

Fig. 5. Behavior of the controlled robot under the two successive set-point changes given by (35). Solid and squared lines represent the behavior for two different control input bounds [see (37) and (39)].

$$
P_{d}(t)= \begin{cases}(6,-3,1)^{T} & \text { for } t \leq 60 s \\ (14,-5,-1)^{T} & \text { for } t>60 s\end{cases}
$$

Figure 5 shows the behavior of the head's coordinates as well as the evolution of the rolling angle $\arcsin \left(p_{\text {rol }}\right)$ for two different control input bounds $\left[\mathbf{u}:=\left(u_{p}, u_{q}, u_{1}, u_{2}, u_{3}\right)\right]$ :

$$
\begin{aligned}
& \mathbf{u}_{\min }=\left(-4^{\circ},-20^{\circ}, 0,-1,0\right) \\
& \mathbf{u}_{\max }=\left(4^{\circ}, 20^{\circ}, 0.5,1,1.4\right)
\end{aligned}
$$

or

$$
\begin{aligned}
& \mathbf{u}_{\min }=\left(-4^{\circ},-10^{\circ}, 0,-1,0\right) \\
& \mathbf{u}_{\max }=\left(4^{\circ}, 10^{\circ}, 0.5,1,0.8\right)
\end{aligned}
$$

The evolution of the control input $\left(u_{p}, u_{q}, u_{1}, u_{2}, u_{3}\right)$ during these scenarios is depicted on figure 6 .
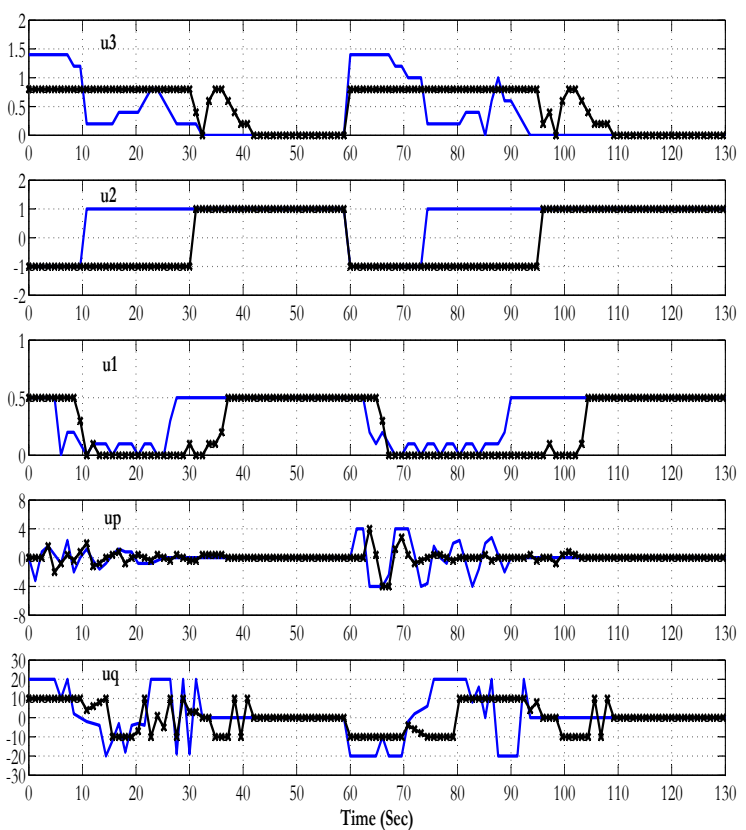

Fig. 6. Evolutions of the control input $\mathbf{u}$ during the scenarios of figure 5 . The solid and squared lines correspond to the constraints (36), (37) and (38), (39) respectively

The evolution of the robot velocity in the earth frame during the same scenarios is presented in figure 7 .

Figures 8 and 9 show the evolution of the different variables in a trajectory tracking scenario tested on the continuous complete model [3]. The desired trajectory is geometrically defined as shown in figure 8 .

\section{CONCLUSION}

In this paper, a complete control scheme for 3D movement of an eel-like robot is proposed. A multi-variable feedback design that enables the tracking of a desired 3D position of the Eel head as well as the stabilization of the rolling angle is presented. This is done using the reduced model that enabled the derivation of a constrained quadratic optimization problem that can be solved on-line in order to better achieve the trajectory tracking as well as the roll angle stabilization tasks. The controller is tested using the reduced and the continuous model [3] for many 3D scenarios. Future work concerns the implementation on the prototype (under construction) as well as the explicit handling of the actuator saturation in terms of 

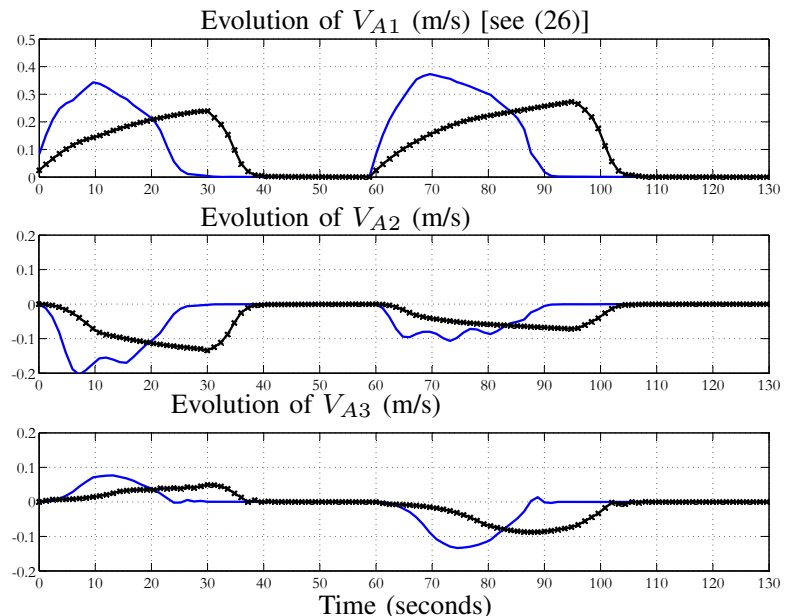

Fig. 7. Evolutions of the robot velocity components in the earth frame during the scenarios of figure 5 .

torque rather than in terms of amplitudes of undulation as it is done in the current paper.

\section{REFERENCES}

[1] M. Alamir, M. El Rafei, G. Hafidi, N. marchand, M. Porez, and F. Boyer. Feedback design for $3 \mathrm{~d}$ movement of an eel-like robot. In Proceedings of the IEEE Int. Conf. Robotics and Automation, pages 256-261, Roma, 2007.

[2] P. R. Bandyopadhyay. Trends in biorobotic autonomous undersea vehicles. IEEE Journal of Oceanic Engineering, 30(1):109-139, January 2005.

[3] F. Boyer, M. Porez, and W. Khalil. Macro-continuous computed torque algorithm for a three-dimensional eel-like robot. IEEE Transaction on Robotics and Automation, 22(4):763-775, August 2006.

[4] J. Carling, T. L. Williams, and G. Bowtell. Self-propelled anguilliform swimming: simultaneous solution of the two-dimensional navierstokes equations and newtons laws of motion. Journal of experimental biology, 201:3143-3166, 1998.

[5] Jack C. K. Chou. Quaternion kinematic and dynamic differential equations. IEEE Transactions on Robotics and Automation, 8(1):5364, February 1992.

[6] J. E. Colgate and K. M. Lynch. Mechanics and control of swimming: A review. IEEE Journal of Oceanic Engineering, 29(3):660-73, July 2004.

[7] M. El Rafei, M. Alamir, N. Marchand, M. Porez, and F. Boyer. Motion control of a three-dimensional eel-like robot without pectoral fins. In Proceedings of IFAC World congress, Seoul, 2008.

[8] R. Mason and J. W. Burdick. Experiments in carangiform robotic fish locomotion. In Proceedings of the IEEE Int. Conf. Robotics and Automation, pages 428-435, San Francisco, 2000.

[9] K. A. McIsaac and J. P. Ostrowski. A geometric approach to anguiliform locomotion modelling of an underwater eel robot. In Proceedings of the IEEE Int. Conf. Robotics and Automation, pages 2843-2848, Detroit, 1999.

[10] K. A. Morgansen, V. Duidam, R. J. Mason, J. W. Burdick, and R.M. Murray. Nonlinear control methods for planar carangiform robot fish locomotion. volume 1, pages 427-434, 2001.

[11] K. A. Morgansen, P. A. Vela, and J. W. Burdick. Trajectory stabilization for a planar carangiform robot fish. In Proceedings of the IEEE Int. Conf. Robotics and Automation, pages 756-762, Washington, 2002.

[12] M. Sfakiotakis, D. M. Lane, and B. C. Davies. Review of fish swimming modes for aquatic locomotion. IEEE Journal of Oceanic Engineering, 24(2):237-252, April 1999.

[13] J. C. Simo. A finite strain beam formulation. the three dimensional dynamic problem. part i: formulation and optimal parametrization. Comp. Meth. Appl. Mech. Eng., 72:276-304, 1989.

[14] M. S. Triantafyllou and G. S. Triantafyllou. An efficient swimming machine. Scientific American, 272:64-70, March 1995.
[15] M. S. Triantafyllou, G. S. Triantafyllou, and R. Gropalkrishnan Optimal thrust development in oscillating foils with application to fish propulsion. J. Fluids Structures, 7:205-224, 1993.

[16] P. A. Vela, K. A. Morgansen, and J. W. Burdick. Underwater locomotion from oscillatory shape deformations. In Proceedings of the 41st IEEE Conference on Decision and Control, pages 2074-2080, Las Vegas, 2002.

[17] J. Yu, L. Wang, and M. Tan. A framework for biomimetic robot fish's design and its realisation. In American Control Conference, pages 1593-1598, Portland, 2005.

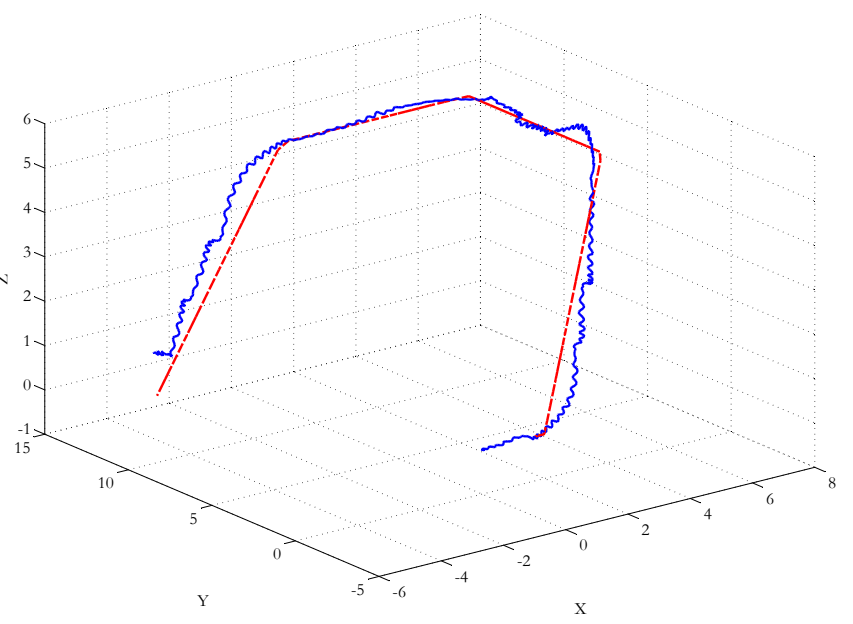

Fig. 8. The $3 D$ trajectory of the eel's head during a 3D trajectory tracking
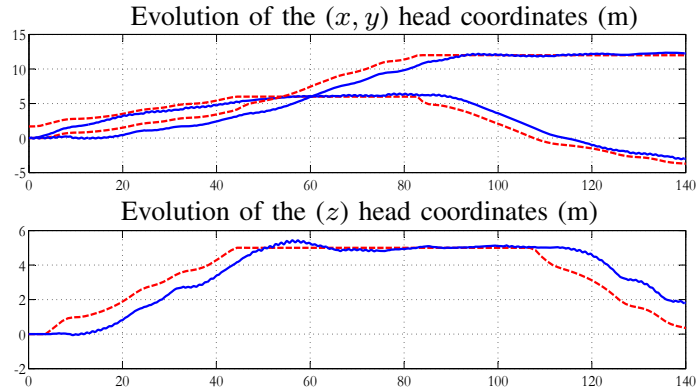

Evolution of the rolling angle $\arcsin \left(p_{r o l}\right)(\mathrm{deg})$

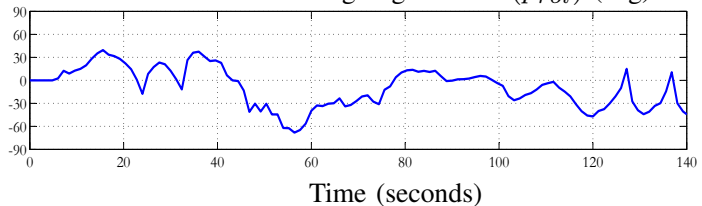

Fig. 9. Behavior of the controlled robot in the scenario of figure 8 . 